\title{
COMPETÊNCIAS PARA FORMAÇÃO DO FISIOTERAPEUTA NO ÂMBITO DAS DIRETRIZES CURRICULARES E PROMOÇÃO DA SAÚDE
}

\section{Kamylla Pereira Borges}

Doutora em Ensino na Saúde (UnB), Docente do Instituto Federal de Ciência e Tecnologia de Goiás (IFG),Câmpus de Anápolis, Brasil.
RESUMO: As competências para promoção da saúde têm sido construídas e validadas no âmbito internacional, destacando-se o projeto CompHP. As Diretrizes Curriculares Nacionais para o curso de fisioterapia estabelecem competências que dialogam com a promoção da saúde. O objetivo desse estudo é relacionar as competências preconizadas pelas Diretrizes Curriculares Nacionais com a promoção da saúde definidas pelo CompHP e identificar essas competências em quatro projetos pedagógicos de cursos de fisioterapia em Goiânia (GO). A metodologia adotada foi análise documental, os dados foram processados com auxílio do software NVivo10. Constatou-se que existem dificuldades para desenvolvimento das competências preconizadas pelas Diretrizes Curriculares Nacionais e para Promoção da Saúde como falta de articulação com a atenção básica do SUS e o currículo com enfoque reabilitador. Concluiu-se que a formação do fisioterapeuta, nesses cursos analisados, ainda está centrada na doença e modelo biomédico, desarticuladas com as competências preconizadas pelas diretrizes e para promoção da saúde.

PALAVRAS CHAVE: Fisioterapia; Promoção da saúde; Educação em Saúde.

\section{COMPETENCES FOR THE FORMATION OF PHYSIOTHERAPISTS WITHIN CURRICULAR GUIDELINES AND HEALTH PROMOTION}

\begin{abstract}
Competences for health promotion have been constructed and validated within international milieu, with special reference to the project CompHP. National Curricular Guidelines for the physiotherapy course establish competences that dialogue with health promotion. Current analysis relates competences recommended by the National Curricular Guidelines with health promotion defined by CompHP and places them within four pedagogical projects of physiotherapy courses in Goiânia GO Brazil. Through documental analyses, data were processed by NVivo10. Several difficulties exist for the development of competences recommended by the National Curricular Guidelines and Health promotion, such as lack of coordination with basic care within the NHS (SUS) and the curriculum focused on rehabilitation. Results show that the formation of the physiotherapist in the courses under study is still centered on disease and the biomedical model, totally disconnected from competences by the guidelines and health promotion.
\end{abstract}

KEY WORDS: Physiotherapy; Health promotion; Health Education. 


\section{INTRODUÇÃO}

A fisioterapia é uma profissão criada sob a tutela dos médicos, historicamente relacionada à reabilitação e readaptação dos movimentos. No século XX, a formação do fisioterapeuta seguiu os moldes da educação médica, com destaque para influência do modelo flexeneriano, orientado para racionalidade científica, atenção médica individual com foco na doença e pouca ênfase para prevenção e promoção da saúde ${ }^{1}$.

A proposição do SUS e o debate acerca do conceito ampliado de saúde na década de 1980 trouxeram à tona a necessidade da mudança no foco de formação dos profissionais, que deixa de ser centrado no atendimento curativo e hospitalocêntrico e passa a se direcionar às várias instâncias de produção da vida e a se ancorar na intersecção dos saberes das ciências sociais e da saúde ${ }^{2}$.

Neste cenário, foram instituídas, por meio da resolução do Conselho Nacional de Educação/Câmara de Educação Superior (CNE/CES) n ${ }^{\circ}$ 04/2002 as Diretrizes Curriculares Nacionais $(\mathrm{DCN})^{3}$ do curso de fisioterapia. Até então, a formação do fisioterapeuta era estruturada de acordo com um currículo mínimo fixado, pelo Conselho Federal de Educação (CFE), em 1983.

As Diretrizes Curriculares Nacionais buscaram romper com a lógica focada na doença e avançaram na direção de uma concepção ampliada de saúde. As DCN trazem como inovação a definição das competências e habilidades para um perfil profissional generalista, humanista, crítico e reflexivo, capaz de atuar em todos os níveis de atenção à saúde (primário, secundário e terciário), visando atender às necessidades do SUS e de saúde da população brasileira ${ }^{4,5}$. Para os cursos de fisioterapia, a formação inclui desenvolver as seguintes competências gerais: atenção à saúde, tomada de decisões baseada em evidências científicas, comunicação, liderança, administração e gerenciamento e educação permanente ${ }^{3}$.

Competência é a capacidade de mobilizar diversos recursos cognitivos para elaboração de estratégias para identificação e resolução de problemas. A competência leva à utilização de várias habilidades e várias habilidades convergem para uma competência ${ }^{6}$.
Ao analisar as competências gerais propostas nas DCN percebe-se que essas vêm ao encontro do domínio de competências de promoção da saúde (PS), que orientam as práticas profissionais na área da saúde em todo o mundo.

A capacitação da força de trabalho para desenvolver e implementar práticas e políticas em promoção da saúde é hoje reconhecida como fundamental para sustentar as ações em promoção de saúde globalmente. Uma iniciativa que vem se destacando como referência é as competências essenciais em promoção da saúde desenvolvidas pelo grupo de trabalho europeu, CompHP ${ }^{7,8,9}$.

O projeto CompHP (Developing Competencies and Professional Standards for Health Promotion Capacity Building in Europe) teve início em 2009 e foi lançado para estabelecer meios e métodos pelos quais o desenvolvimento de competências para qualificação da força de trabalho no âmbito da PS possam ser implementadas ${ }^{7}$. As principais competências elencadas são o favorecimento de mudanças, a advocacia para saúde, as parcerias, a comunicação, a liderança, o diagnóstico, o planejamento, a implementação, a avaliação e a pesquisa ${ }^{7}$. Alguns países da União Europeia já estão usando as recomendações do projeto CompHP para orientar reformas na educação profissional em saúde pública? .

O debate em torno das competências para formação do fisioterapeuta é importante, pois atualmente existe um processo de mudança no modelo de formação na saúde, e pesquisas demonstram que apesar de hoje a atuação do fisioterapeuta abranger os três níveis de atenção à saúde (primário, secundário e terciário), as origens da fisioterapia, enquanto profissão relacionada à reabilitação da doença ${ }^{10}$, trazem desafios para 0 desenvolvimento das competências preconizadas pelas DCN e para atuação na promoção da saúde. Muitos cursos de graduação ainda mantêm uma visão restrita da atuação do fisioterapeuta, com ensino voltado para o tratamento de sequelas, o que dificulta o entendimento do estudante sobre a atuação para promoção da saúde ${ }^{11-15}$.

Assim, com o presente estudo pretendeu-se relacionar as competências gerais preconizadas pelas DCN com as competências para a promoção da saúde 
definidas pelo CompHP e identificálas nos PPC dos cursos de graduação em fisioterapia da cidade de GoiâniaGO. O estudo é relevante, pois traz apontamentos que contribuem para reflexão acerca da redefinição da formação em fisioterapia, focando a necessidade de ampliação e diversificação da sua atuação na saúde, possibilitando novas discussões em torno da formação em fisioterapia para a promoção da saúde e de acordo com as DCN.

\section{MÉTODO}

Esse trabalho é fruto da tese de doutorado "As competências para formação do fisioterapeuta na perspectiva das DCN e da Promoção da Saúde", defendida pelo projeto Pró-Ensino na Saúde, da UnB e foi aprovado pelo Comitê de Ética em Pesquisa da Faculdade de Ciências da Saúde da Universidade de Brasília (projeto 186/12). Trata-se de um estudo de natureza qualitativa, do tipo exploratório e descritivo realizado por meio da análise documental. A pesquisa foi realizada na cidade de Goiânia/GO de novembro de 2014 a junho de 2015, em Instituições de Ensino Superior (IES) que ofertavam cursos de graduação em fisioterapia. A análise documental considerou os PPC das IES participantes, as DCN/Fisio e as competências para promoção da saúde definidas pelo CompHP. O universo da pesquisa se constituiu de oito IES; para seleção da amostra foram considerados dois critérios de inclusão: 1) permitir o acesso ao Projeto Pedagógico de Curso (PPC); 2) aceitar participar da pesquisa, assinando o Termo de Concordância. Aquelas que não contemplaram os critérios de inclusão foram excluídas do estudo.

Quatro IES atenderam aos critérios de inclusão, para preservar a identidade das instituições; essas foram nomeadascomo Fac 1, Fac 2, Fac 3, Fac 4.

O PPC foi escolhido como um dos documentos a ser analisado, pois se constitui como organizador e consolidador da programação das atividades acadêmicas dos cursos de graduação, definindo os perfis profissionais que se pretende formar ${ }^{16}$. Os PPC dos cursos de fisioterapia foram disponibilizados na forma digital pelas instituições participantes.

Para realização da análise documental foi utilizado como instrumento de coleta de dados um roteiro previamente estabelecido. Contendo os seguintes aspectos:

1- caracterização dos cursos: modalidade de oferta, turno, regime, duração média prevista, carga horária e número de vagas ofertadas;

2- competências a serem desenvolvidas listadas pelo curso;

3- matriz curricular: organização do currículo, distribuição da carga horária entre as disciplinas; o ementário e os objetivos das disciplinas. Atividades complementares, estágios e campos de estágio.

Os dados da pesquisa foram processados com o auxílio do software NVivo 10, que oportunizou a criação das categorias para análise de conteúdo, definidas posteriormente a partir do agrupamento dos dados ${ }^{17}$.

As categorias de análise foram definidas tomando como referência as competências gerais DCN/Fisio, os Domínios de Competências da Promoção da Saúde e os conteúdos que emergiram dos documentos. Assim, foram identificadas, com auxílio do NVivo, unidades de registro: frases, palavras ou termos, que estavam voltados para o desenvolvimento das competências e essas foram consideradas como categorias de análise e possibilitaram a relação entre os aspectos do roteiro utilizados para análise documental e o desenvolvimento das competências já especificadas.

\section{RESULTADOS}

Esse tópico foi dividido em duas partes, na primeira realizamos a análise da relação entre as competências gerais, preconizadas pelas DCN e as para promoção da saúde de acordo com o CompHP, na segunda identificamos as competências nos PPC dos cursos de graduação em fisioterapia da cidade de Goiânia-GO. 
RELAÇÃO ENTRE AS COMPETÊNCIAS PRECONIZADAS PELAS DCN E AS COMPETÊNCIAS PARA ATUAÇÃO NA PROMOÇÃO DA SAÚDE

A estruturação das competências e habilidades designa um conjunto de qualificações, conhecimentos, valores e atitudes que fundamentarão a atuação profissional. $\mathrm{O}$ art. $4^{\mathrm{O}}$ das DCN/Fisio define seis competências e habilidades gerais para o exercício da profissão de fisioterapeuta:

1) atenção à saúde, que se refere à atuação em todos os níveis de saúde de forma integrada e contínua, tanto em nível individual quanto coletivo;

2) tomada de decisões para avaliar, sistematizar e decidir condutas adequadas baseadas em evidências científicas;

3) comunicação, que propicia a interação entre profissionais e comunidade;

4) liderança pautada no trabalho em equipe multiprofissional com responsabilidade e empatia para tomada de decisões;

5) administração e gerenciamento, tanto da força de trabalho quanto recursos físicos e materiais;

6) educação permanente que favorece a aprendizagem contínua ${ }^{3}$. Extraímos os conceitos dessas competências e relacionamos com as competências de promoção da saúde preconizadas pelo CompHP, visando identificar possíveis convergências entre elas.

A competência geral de atenção à saúde é uma competência fundamental para operacionalização do princípio da integralidade do SUS e está diretamente relacionada ao valor ético definido pelo CompHP que se refere a visar todos o determinantes do processo saúde doença ${ }^{3,7}$.

A consciência sobre a importância dos determinantes sociais na saúde e sobre a necessidade do combate às iniquidades de saúde por eles geradas é fundamental para atuação de acordo com pressupostos da promoção da saúde. Esse conhecimento permite o desenvolvimento de ações voltadas para mudanças nos modelos assistenciais e para a ampliação da autonomia dos sujeitos, incluindo intervenções nas condições socioeconômicas, ambientais e culturais ${ }^{18}$.

Tomada de decisões está dentro dos valores éticos para PS definidos pelo CompHP como a busca por informações e evidências apropriadas na implementação de ações que influenciem a saúde. Ao aprofundar os conhecimentos no campo da saúde, de forma geral, o profissional se torna apto para enfrentar as mais variadas situações em seu ambiente de trabalho e tomar as decisões mais acertadas em relação a sua atuação profissional e a saúde tanto em nível individual e coletivo ${ }^{3,7}$.

A comunicação é uma competência geral preconizada tanto pelas DCNs quanto pelo CompHP. Diz respeito à comunicação verbal, não verbal e habilidades de escrita e leitura, além do domínio de línguas estrangeiras e tecnologias de informação e comunicação. Da mesma forma, a liderança é referida tanto nas DCN/ Fisio quanto no projeto CompHP e, em ambas, ela diz respeito ao desenvolvimento da capacidade de trabalho e aprendizagem em equipe multiprofissional, o que requer compromisso, habilidades próprias de comunicação e de gerenciamentos de processos e gestão de pessoas, tendo como foco o bem-estar individual e coletivo ${ }^{3,7}$.

NoprojetoCompHP,acompetênciaAdministração e Gerenciamento está inclusa na competência Liderança e em Planejamento e Implementação. O planejamento se refere ao desenvolvimento de planos de ação para serem executados de acordo com os recursos, parceiros e metas da PS. A implementação está voltada para o gerenciamento dos recursos necessários para implementação das ações planejadas e o monitoramento do plano de ação, além do desenvolvimento, condução e utilização dos recursos e materiais de forma apropriada ${ }^{7}$. Assim sendo, as competências denominadas planejamento e implementação se complementam e correspondem as de administração e gerenciamento referidas nas DCN/Fisio.

Por último, temos a Educação Permanente, que se volta à aprendizagem contínua dos profissionais. De acordo com Ceccim, ${ }^{19}$ a educação permanente foi criada para dimensionar, e não prolongar o tempo de formação ou carreira dos profissionais. Ela se dá na interlocução entre gestão, atenção e participação nas áreas específicas de saberes e práticas da saúde, com entrosamento na área da educação e atenção à saúde e se constitui 
como o caminho mais adequado para empreender as transformações no trabalho na área da saúde.

A educação permanente está relacionada aos seguintes domínios de competências do projeto CompHP: parceria, diagnóstico e avaliação e pesquisa. A parceria diz respeito ao trabalho em colaboração com áreas de conhecimento e setores diferentes para aumentar o impacto e a sustentabilidade das ações em promoção da saúde 7 . A educação permanente, ao preconizar a interlocução dos saberes empreendidos nos ambientes acadêmicos, na gestão e na atenção em saúde propriamente dita, também preconiza o desenvolvimento de parcerias, pois sem elas não seria possível trabalhar ou obter uma formação que abrangesse instituições formadoras e os serviços de saúde em suas várias esferas de gestão.

A formação para desenvolver a competência da educação permanente deve buscar atender as necessidades de saúde das pessoas e da populaçãa ${ }^{20}$, o que dialoga com a proposta de desenvolvimento da competência diagnóstico, informação e planejamento preconizadas pelo CompHP.

Quando o projeto se refere a diagnóstico, quer dizer diagnosticar as necessidades de saúde da sociedade no contexto dos determinantes do processo saúde doença, sejam eles políticos, econômicos, sociais, culturais, ambientais, comportamentais ou biológicos que promovam ou comprometam a saúde ${ }^{7}$. Nessa competência, a questão da necessidade de visar todos os determinantes do processo saúde doença é fundamental, pois é na capacidade de realizar o diagnóstico das necessidades e potencialidades, em termos de saúde dos sujeitos e coletividades, que o conhecimento sobre os determinantes sociais se torna imprescindível.
O desenvolvimento dessas três competências elencadas pelo CompHP (parceria, diagnóstico e avaliação e pesquisa) implicaria na concretização da educação permanente em saúde como uma dimensão de competência mais abrangente na formação do fisioterapeuta pois, de acordo com Ceccim $^{19}$, colocar a educação permanente para saúde em evidência para a formação e para o SUS requer "trabalhadores que aprendam a aprender; práticas cuidadoras; intensa permeabilidade ao controle social; compromissos de gestão com a integralidade; desenvolvimento de si, dos colegas, institucional e político da saúde".

Diante do exposto, abaixo demonstramos resumidamente a relação existente entre as competências gerais, preconizadas pelas DCN, lado esquerdo, e as para promoção da saúde definidas pelo CompHP, lado direito:

- atenção à saúde-diagnóstico de necessidades;

- tomada de decisões - avaliação; pesquisa;

- comunicação - comunicação;

- liderança - liderança;

- administração e gerenciamento planejamento e implementação;

- educação permanente - parceria; diagnóstico; avaliação; pesquisa

\section{AS COMPETÊNCIAS IDENTIFICADAS NOS PPC DOS CURSOS ANALISADOS}

O Quadro 1 sistematiza os dados referentes à carga horária, ao turno, ao regime e à duração dos cursos de cada IES participante da pesquisa.

Quadro 1. Caracterização dos cursos de fisioterapia de acordo com os PPCs

\begin{tabular}{|l|c|c|c|c|}
\hline ITENS & Fac 1 & Fac 2 & Fac 3 & Fac 4 \\
\hline Modalidade de oferta & Presencial & Presencial & Presencial & Presencial \\
\hline Turno & Integral & Matutino & Integral & Noturno \\
\hline Regime & Crédito-semestral & Crédito- semestral & Seriado- semestral & Seriado- semestral \\
\hline Duração média prevista & 9 semestres & 9 semestres & 10 semestres & 8 semestres \\
\hline Carga horária & 4350,55 & 4030 & 4005 & 4200 \\
\hline Número de vagas anuais & 30 & 110 & 100 & 50 \\
\hline
\end{tabular}


De acordo com a art. $6^{\circ}$ das DCN/Fisio, a formação do fisioterapeuta deve estar fundamentada em quatro blocos de conteúdos: ciências biológicas e da saúde (CBS), ciências sociais e humanas (CSH), conhecimentos biotecnológicos (CBT) e conhecimentos fisioterapêuticos $(\mathrm{CF})^{3}$.

Todos os PPC analisados trouxeram na íntegra as seis competências e habilidades gerais preconizadas pelas
DCN/Fisio. Além disso, a análise da matriz curricular dos cursos pesquisados evidenciou que todos eles organizam os conteúdos de acordo com os blocos de conhecimento preconizados pelas DCN. Além dos conteúdos destacados pelas diretrizes, a Fac1 traz em sua matriz um bloco de conteúdos a mais: Conhecimentos Profissionalizantes (CP), no qual se encontram agrupados os estágios supervisionados do bloco de CF.

Quadro 2. Distribuição de carga horária por bloco de conhecimento em cada instituição pesquisada

\begin{tabular}{|l|c|c|c|c|c|c|c|}
\hline \multicolumn{1}{|c|}{ INSTITUIÇÕES } & CBS & CSH & CBT & CF & CP & AC & Total \\
\hline Fac 1 & 849,91 & 339.96 & 169,99 & 1926,46 & 1062,51 & 200 & $4,548,83$ \\
\hline Fac 2 & 1200 & 390 & 470 & 1770 & - & 200 & 4030 \\
\hline Fac 3 & 900 & 270 & 230 & 1635 & 810 & 180 & 4005 \\
\hline Fac 4 & 800 & 440 & 600 & 2360 & - & 160 & 4200 \\
\hline
\end{tabular}

Ao comparar a carga horária contida em cada grupo, percebemos a discrepância existente entre as áreas do conhecimento. O bloco de conhecimentos fisioterapêuticos (CF) é predominante na matriz curricular de todas as instituições e varia de $41 \%$ a $56 \%$ da carga horária total dos cursos, seguido pelos conhecimentos biológicos e da saúde (18 a 29\%) e conhecimentos profissionalizantes (23\% da Fac1 e 21\% da Fac 3). Os conhecimentos das áreas sociais e humanas variaram de 7 a $10 \%$ e de $4 \%$ a $14 \%$, quando analisados os conhecimentos biotecnológicos.

No Quadro 3 são demonstradas as disciplinas com conteúdos diretamente relacionados ao SUS em cada IES pesquisada.

Quadro 3. Disciplinas com conteúdos diretamente relacionados ao SUS e suas respectivas cargas horárias $(\mathrm{CH})$

\begin{tabular}{|c|c|c|c|c|c|c|c|}
\hline \multicolumn{2}{|l|}{ Fac 1} & \multicolumn{2}{|l|}{ Fac 2} & \multicolumn{2}{|l|}{ Fac 3} & \multicolumn{2}{|l|}{ Fac 4} \\
\hline Disciplina & $\mathrm{CH}$ & Disciplina & $\mathrm{CH}$ & Disciplina & $\mathrm{CH}$ & Disciplina & $\mathrm{CH}$ \\
\hline $\begin{array}{l}\text { Legislação e políticas } \\
\text { de saúde }\end{array}$ & 28,33 & Saúde e meio ambiente & 30 & $\begin{array}{l}\text { Saúde e meio } \\
\text { ambiente }\end{array}$ & 45 & Epidemiologia & 40 \\
\hline $\begin{array}{l}\text { Fisioterapia } \\
\text { comunitária, } \\
\text { preventiva e social }\end{array}$ & 56,66 & Saúde pública & 60 & $\begin{array}{l}\text { Legislação e } \\
\text { políticas de saúde }\end{array}$ & 45 & Saúde pública & 40 \\
\hline \multirow[t]{3}{*}{$\begin{array}{l}\text { Administração e } \\
\text { serviços de saúde }\end{array}$} & 28,33 & $\begin{array}{l}\text { Fisioterapia na } \\
\text { comunidade }\end{array}$ & 60 & $\begin{array}{l}\text { Fisioterapia em } \\
\text { saúde pública }\end{array}$ & 45 & $\begin{array}{l}\text { Programa de saúde da } \\
\text { família }\end{array}$ & 80 \\
\hline & & $\begin{array}{l}\text { Administração serviços } \\
\text { de saúde }\end{array}$ & 60 & & & & \\
\hline & & $\begin{array}{l}\text { Estágios } \\
\text { supervisionados em } \\
\text { fisioterapia em saúde } \\
\text { pública }\end{array}$ & 150 & & & & \\
\hline
\end{tabular}


A instituição que apresenta em sua matriz curricular a maior carga horária de disciplinas voltadas para atuação e conhecimento do SUS de forma direta é a Fac 2. As demais IES apresentam um conjunto de disciplinas semelhantes no que diz respeito aos conhecimentos em saúde pública e o SUS, privilegiando $\mathrm{o}$ aspecto teórico.

Fac 1, Fac 3 e Fac 4 não deixam claro, em seus PPC, a orientação da formação de seus cursos em fisioterapia de acordo com o SUS, conforme preconizado pelas DCN/ Fisio. Havendo menção ao SUS somente como parte do conteúdo das disciplinas listadas acima.

Já Fac 2 indica em seu PPC que foi realizada uma reformulação curricular visando "atender às demandas provindas do contexto social e também de exigências legais à nova Lei de Diretrizes e Bases e à Política do SUS", em uma demonstração de preocupação em formar os futuros fisioterapeutas de acordo com os princípios e diretrizes do SUS.

Nos PPC da Fac 1, Fac 3 e FAc 4, não identificamos as competências de parceria e diagnóstico $\mathrm{e}$, consequentemente, as de educação permanente também não foram localizadas, uma vez que esta só pode se concretizar mediante o desenvolvimento desses dois domínios, pois sem parceria com as redes de atenção à saúde não pode haver diagnóstico de necessidades, sem os quais não há educação permanente. Percebemos o estímulo à educação continuada, que difere da educação permanente, pois educação continuada está relacionada ao aperfeiçoamento da carreira profissional geralmente por meio da educação formal visando o mercado de trabalho. No caso específico das instituições analisadas há o estímulo à educação continuada com foco nas especialidades em projetos de pesquisa, extensão e eventos pontuais.

Em relação às demais competências: comunicação, tomada de decisões e avaliação e pesquisa, estas foram identificadas nos PPC dos quatro cursos. A Fac1 e Fac 2 tentam trabalhar a competência liderança por meio de estratégias pontuais que não abrangem todos os estudantes, mas apenas aqueles envolvidos nas Ligas Acadêmicas ou Centros Acadêmicos. A liderança, como domínio de competência para promoção da saúde, está relacionada à utilização de habilidades para promover empoderamento, a participação da comunidade e, também, para incentivar o trabalho em equipe ${ }^{7}$. Essas habilidades e as suas formas de mobilização não foram identificadas em nenhum dos PPC analisados.

A Fac 1, Fac 2 e Fac 4 buscam mobilizar a competência administração e gerenciamento, e sua correspondente na promoção da saúde, planejamento e implementação, por meio da oferta da disciplina administração de serviços de saúde, cuja ementa se assemelha a de todas as demais instituições analisadas, na qual está enfatizada as formas de organização e ( administração do sistemas público e privado de saúde brasileiro, a fim de preparar o estudante para atuar na gestão de serviços de saúde.

A parceria é fundamental para o desenvolvimento da educação permanente como competência dos futuros fisioterapeutas. De acordo com Dempsey et $\mathrm{al}^{7}{ }^{7}$ ela envolve o trabalho colaborativo com diversas áreas do conhecimento, disciplinas, setores e redes de trabalho. Pressupõe o ensino interdisciplinar a articulação com os locus de trabalho na área da saúde e com os atores envolvidos: comunidade, profissionais, gestores.

$\mathrm{O}$ desenvolvimento da parceria leva ao terceiro domínio de competência associada à educação permanente: diagnóstico. O diagnóstico está associado às necessidades das pessoas, da população e das comunidades, no contexto dos determinantes políticos, econômicos, sociais, culturais e ambientais. O estudante só poderá desenvolver essa competência se forem desenvolvidas parcerias com os diversos atores envolvidos nos sistemas de saúde.

Nos PPC da Fac 1, Fac 3 e FAc 4 não identificamos as competências de parceria e diagnóstico e, consequentemente, as de educação permanente também não foram localizadas, uma vez que esta só pode se concretizar mediante o desenvolvimento desses dois domínios.

\section{DISCUSSÃO}

De acordo com os resultados apresentados, observa-se que não existe equilíbrio entre as diferentes áreas do saber, como preconizado pelo art. 13 , inciso 
II das Diretrizes. Os currículos das quatro instituições ainda estão majoritariamente fundamentados nos conhecimentos biológicos e da saúde e no conhecimento técnico especializado da fisioterapia.

Os currículos da Fac1, Fac 3 e Fac 4 seguem o modelo tradicional de formação e se mostram ainda influenciados pelos ciclos básicos, pré-profissionalizantes e profissionalizantes do currículo mínimo (resolução CFE $\left.\mathrm{n}^{0} 4 / 1983\right)^{21}$ com predomínio de disciplinas compartimentadas com pouca integração entre as mesmas. No primeiro ano do curso estão as disciplinas voltadas para os conhecimentos básicos em biologia e saúde; no segundo ano, os conhecimentos técnicos da fisioterapia, e nos últimos anos os conhecimentos voltados para fisioterapia aplicada. O foco está no biológico, na fragmentação das disciplinas e nas diferentes especialidades da fisioterapia.

Essa estrutura curricular não é exclusiva dos cursos analisados. De acordo com Albuquerque et $\mathrm{a}^{22}$, a maioria dos cursos da área da saúde possui currículos organizados em disciplinas, nos quais os ciclos profissionais e básicos são separados ou têm pouca relação entre si. Os autores destacam, também, a fragmentação dos conteúdos e a supervalorização das disciplinas voltadas para os aspectos biológicos, em detrimento daquelas voltadas para as humanidades, como elementos que contribuem para que, depois de formados, os profissionais direcionem suas práticas para a especialização supervalorizando os aspectos biológicos no processo saúde-doença.

Afragmentação em disciplinaslevaàfragmentação da compreensão do ser humano, reduzindo a própria existência humana à objetividade e o cuidado em saúde à assistência voltada para abordagem biologicista ${ }^{23,24}$. O que dificulta a efetivação de uma formação ancorada no princípio da integralidade.

Na Fac 2 verificamos um enfoque diferente, na matriz curricular não há disciplinas voltadas para as áreas de especialização da fisioterapia. A partir do quinto e sexto períodos, ao invés das disciplinas relacionadas à fisioterapia aplicada, vemos: fisioterapia na comunidade, fisioterapia na saúde da criança e adolescente, fisioterapia na saúde do idoso, fisioterapia na saúde da mulher e fisioterapia na saúde do adulto.
As ementas de cada uma dessas disciplinas demonstram a articulação entre avaliação, diagnóstico funcional, prevenção e reabilitação. Ao invés do foco na especialidade e sua aplicação, ele está na saúde de modo integral, e os estudantes são estimulados a enxergar não a patologia, mas o ser humano: criança, adolescente, mulher, homem ou idoso.

Essa abordagem curricular representa um avanço na formação do fisioterapeuta em Goiás no que concerne a adequação às DCN/Fisio e a articulação da formação aos princípios do SUS e promoção da saúde. Uma prática fundamentada na integralidade deve saber articular a oferta de ações ou procedimentos preventivos com procedimentos assistências, considerando o contexto particular de cada indivíduo ${ }^{25}$. Essa estruturação curricular favorece essa prática aos estudantes, pois estes são estimulados a enxergar o ser humano desde uma perspectiva integral e articular os conhecimentos de todos os campos da fisioterapia para definir o plano de cuidado para esse indivíduo.

Outro diferencial do currículo da Fac 2 é o estágio supervisionado em fisioterapia na comunidade e fisioterapia na saúde pública. O objetivo dos estágios nessas disciplinas, de acordo com o PPC do curso, é consolidar a prática dos profissionais para o SUS e associar ações assistenciais e educativas com ênfase na promoção da saúde.

Nessa instituição, o SUS é um dos cenários de práticas e de aprendizagem. Privilegiar o SUS como cenário de aprendizagem ocasiona a reflexão sobre a realidade da saúde brasileira e favorece a transformação e consolidação dos modelos de atenção à saúde, pautados pelos valores do SUS $^{22}$.

Profissionais que durante a formação são inseridos nas redes de atenção básica e que conhecem o SUS e as políticas de saúde têm mais chances de assumir um compromisso em sua atuação profissional diante das necessidades do SUS, o que contribui para efetivar a transformação das práticas profissionais e organização do trabalho em saúde ${ }^{27}$.

Luchese et $\mathrm{a}^{28}$ apontam o distanciamento entre a formação profissional e as necessidades concretas do SUS o que indica ser preciso uma reorientação da formação. Para os pesquisadores, no processo de formação deve- 
se trabalhar as seguintes dimensões: teórica, cenários de prática e orientação pedagógica; essas dimensões devem perpassar os determinantes de saúde e doença, educação permanente, integração ensino-serviço, integração dos serviços das IES com os serviços de saúde, análise crítica dos serviços e aprendizagem ativa.

Quanto a competência relacionada à Atenção à Saúde, pelos currículos com formado tradicional, privilegiando a fisioterapia aplicada e o aspecto reabilitador da profissão, essa competência não foi identificada na Fac 1, Fac 3 e Fac 4. As práticas são desenvolvidas em ambientes clínicos e hospitalares em sua maioria, não havendo indicação de parcerias com a rede de atenção básica, de forma que os estudantes acabam forçados a visualizar a fisioterapia apenas em seu caráter curativo, e não preventivo o que dificulta o desenvolvimento de uma atuação pautada na promoção da saúde.

Já na Fac 2 essa competência é passível de ser desenvolvida pela estruturação de seu currículo, com foco no ser humano integral e articulação dos três níveis de atenção nas ementas das disciplinas.

Em relação à educação permanente, verificamos que as quatro instituições têm diversas formas de estimular a pesquisa, sendo um pressuposto bastante valorizado. As IES incentivam a educação continuada e a pesquisa por meio de programas de iniciação científica, projetos de extensão e da realização de eventos científicos.

Mas, essas estratégias por si só não podem ser consideradas eficientes para se desenvolver a competência educação permanente, nos termos preconizados pelas DCN/Fisio, porque ela é uma competência associada à formação para atuação no e para o SUS. De acordo com Ceccim e Feuerwerker, ${ }^{20}$ a educação permanente pressupõe uma aprendizagem significativa para transformação das práticas profissionais. Ou seja, é preciso que haja integração entre ensino e a rede de atenção básica no SUS, favorecendo, de acordo com os autores, o quadrilátero ensino, gestão, atenção e controle social.

O artigo $5^{\circ}$ das DCN/Fisio destaca que "a formação do fisioterapeuta deverá atender ao sistema de saúde vigente no país"3. Articular a formação do fisioterapeuta e o SUS é importante para o desenvolvimento das competências gerais e para a promoção da saúde, pois existe um diálogo entre o SUS e a promoção de saúde, tendo em vista, especificamente o enriquecimento da formulação de políticas de saúde no Brasil, resgatando a concepção ampliada de saúde adotada pelo movimento da reforma sanitária ${ }^{26}$. Desse modo, incorporar ambientes de aprendizagem no SUS contribui para consolidação e desenvolvimento das competências preconizadas tanto pelas DCN como para atuação na promoção da saúde.

Os desafios para a formação do profissional fisioterapeuta ainda são muitos, especialmente no que se refere ao desenvolvimento de competências e habilidades gerais para o trabalho em uma perspectiva para atender às necessidades de saúde da população, levando em conta o conceito ampliado de saúde, no que tange ao trabalho em equipe multidisciplinar e interprofissional, a integralidade das ações em saúde, e atuação em todos os níveis de atenção.

No presente estudo verificamos que as DCN/ Fisio são utilizadas como referencial para elaboração dos PPC dos cursos analisados, mas não encontramos informações suficientes no documento para afirmar que as IES conseguem desenvolver as competências gerais por elas elencadas, tampouco as competências voltadas para atuação na promoção da saúde. A formação evidenciada permanece focada no paradigma biologicista e no currículo mínimo de 1983, exceto pela Fac 2, que tenta estimular uma formação pautada na integralidade por meio de disciplinas com foco na saúde integral dos indivíduos e não nas especialidades da fisioterapia.

O currículo com enfoque reabilitador da Fac1, Fac 3 e Fac 4, constatado na análise documental dos PPC, contribui para dificultar o desenvolvimento das competências para promoção da saúde, pois favorece a manutenção da concepção biomédica de saúde, fundamentada no modelo de ensino tradicional, direcionado para as especialidades da fisioterapia.

Assim sendo, uma das principais dificuldades encontradas para adequação da formação das DCN e desenvolvimento das competências gerais preconizadas pelas diretrizes e as relacionadas à atuação do fisioterapeuta na PS foi a inexistência ou pouca articulação do ensino com a rede de atenção básica do SUS e o currículo voltado para as especialidades da fisioterapia. 


\section{CONCLUSÃO}

As informações e reflexões aqui apresentadas são importantes, pois, podem fundamentar o planejamento de estratégias para reorientação da formação do fisioterapeuta. As ações para reorientação da formação terão mais probabilidades de serem efetivadas se os sujeitos centrais nela envolvidos, docentes, alunos e fisioterapeutas tiverem a compreensão das causas das lacunas da formação em fisioterapia enfrentadas atualmente.

Embora os resultados do estudo realizado não possam ser generalizados dadas as características e especificidades regionais das realidades onde as instituições participantes estão inseridas, cujas singularidades precisam ser levadas em conta, o trabalho traz elementos para suscitar reflexões e que contribuem para fundamentar discussões que possam servir para a reorientação da formação em fisioterapia, que seja mais articulada ao sistema de saúde vigente no país e às tendências mundiais de maior integralidade de atenção à saúde.

As dificuldades apontadas sinalizam para a necessidade de realização de novos estudos, no sentido de se buscar medidas que possam contribuir para ruptura do paradigma de formação vigente em fisioterapia e avancem para consolidar práticas voltadas para a saúde coletiva e para a promoção da saúde da população.

\section{REFERÊNCIAS}

1. Pagliosa FL, Daros MA. O Relatório de Flexner: para o bem e para o mal. Rev Bras Educ Méd. 2008;4(32), 492-9.

2. Ceccim RB. Invenção da saúde coletiva e do controle social em saúde no Brasil: nova educação na saúde e novos contornos e potencialidades à cidadania. REU. 2007;33(1),29-48.

3. Ministério da Educação. Conselho Nacional de Educação. Câmara de Educação Superior. Resolução CNE/CES 4 de 19 de fevereiro de 2002. Institui Diretrizes Curriculares Nacionais do curso de graduação em Fisioterapia. Brasília: Diário Oficial da
União, Seção 1, p. 11.

4. Almeida MJ, Campos JJB, Turini B, Nicoletto SCS, Pereira LA, Rezende LR, Mello PL. Implantação das Diretrizes Curriculares Nacionais na graduação em Medicina no Paraná. Rev Bras Educ Méd. 2007; 31(2):156-65.

5. Fernandes JD, Rebouças LC. Uma década de Diretrizes Curriculares Nacionais para a Graduação em Enfermagem: avanços e desafios. Rev Bras Enferm. 2013;66(spe):95-101.

6. Perrenoud P. 10 novas competências para ensinar. Porto Alegre: Artmed; 2000

7. Dempsey C, Barry M, Battel-Kirk B. The CompHP core competencies framework for health promotion handbook: workpackage 4. Galway: Executive Agency for Health Promotion and Consumers: National University of Ireland, 2011. [internet] Disponível em: <http://www.iuhpe.org/images/PROJECTS/ ACCREDITATION/CompHP_Competencies_ Handbook.pdf $>$. Acesso em: 10 jun. 2015.

8. Barry MM, Allegrante JP, Lamarre MC, Aild ME, Taub A. Galway consensus conference: international collaboration on the development of core competencies for health promotion and health education. IUHPE. Global Health Promotion. 2009; 16(2):42-54.

9. Allegrante JP, Barru MM, Auld ME, Lamarre MC. Galway revisited: tracking global progress in core competencies and quality assurance for health education and health promotion. Health Education \& Behavior. 2012;39(6):643-7.

10. Rebelatto JR, Botomé SP.Fisioterapia no Brasil: Fundamentos para uma ação preventiva e perspectivas profissionais. São Paulo: Manole; 1999.

11. Medeiros PA, Foletto PHM, Mayer MS. Contribuições da Visita Domiciliar na Formação em Fisioterapia. Trab. Educ. Saúde (Rio de Janeiro). 2012;10(3):40726.

12. Formiga NFB, Ribeiro KSQS. Inserção do 
Fisioterapeuta na Atenção Básica: Uma Analogia entre Experiências Acadêmicas e a Proposta dos Núcleos de Apoio a Saúde da Família (NASF). Rev Bras Ciênc Saúde. 2012;16(2):113-22.

13. Rodriguez MR. Análise histórica da trajetória profissional do fisioterapeuta até sua inserção nos Núcleos de Apóio a Saúde da Família (NASF). Comun. Ciências Saúde. 2010;21(3):261-6.

14. Araújo FRO. Discursos e práticas na formação de profissionais fisioterapeutas: realidade de cursos de Fisioterapia na região nordeste do Brasil. [Dissertação]. Natal (RN): Universidade Federal do Rio Grande do Norte; 2009.

15. Silva DJ, Daros MA. Inserção de profissionais de Fisioterapia na equipe de saúde da família e Sistema Único de Saúde: desafios da formação. Ciênc. Saúde Coletiva. 2007;12(6):1673-81.

16. Masetto MT. Competência Pedagógica do Professor Universitário. São Paulo: Summus; 2012.

17. QSR International. NVivo 10 for Windows: manual. [internet]. Acesso em 10 de maio de 2015. Disponível em http://download.qsrinternational. com/Document/NVivo10/NVivo10-Getting-Started Guide-Portuguese.pdf

18. Carvalho AI, Buss PM. Determinantes Sociais na Saúde, na Doença e na Intervenção. In:. Giovanella L, Escorel S, Lobato IVC, Noronha JC, Carvalho AI, organizadores. Políticas e Sistema de Saúde do Brasil. Rio de Janeiro: Fiocruz; 2013, p. 141-166.

19. Ceccim RB. Educação Permanente em Saúde: descentralização e disseminação de capacitação pedagógica na saúde. Ciênc. Saúde Coletiva. 2005; 10(4):975-86.

20. Ceccim RB, Feuerwerker LO. Quadrilátero da formação para a área da saúde: ensino, gestão,atenção e controle social. Physis. 2004;14(1):41-65.

21. Ministério da Educação. Conselho Federal de Educação. Resolução 4. Fixa os mínimos de conteúdos e duração dos cursos de fisioterapia e terapia ocupacional [internet]. Brasília (DF); 28 de fevereiro de 1983 [acesso em 15 de junho de 2015]. Disponível em: http://www.prolei.inep.gov. br/pesquisar.do;jsessionid $=$ A05389F814EE4C24BA 603F755C800E7D? codThesaurus $=37179$.

22. Albuquerque VS, Batista RS, Tanji S, Moço ETM. Currículos disciplinares na área de saúde: ensaio sobre saber e poder. Interface (Botucatu). 2009; 13(31):261-72.

23. Garcia MAA, Pinto AIBCS, Odoni APC, Longhi BS, Machado LI, Linek MDS, Costa NA. Interdisciplinaridade e Integralidade no ensino em saúde. Rev. Cienc. Med. 2006;15(6):473-85.

24. BispoEPF, Tavares CHF, TomazJMT. Interdisciplinarity in healthcare education: the preceptor's view of family health. Interface (Botucatu). 2014;18(49):33750.

25. Mattos R. A. A integralidade na prática (ou sobre a prática da integralidade). Cad. de Saúde Pública. 2004;20(5):1411-16.

26. Teixeira CF, Solla JP. Modelo de atenção à saúde: vigilância e saúde da família. Salvador: EDUFBA; 2006.

27. Araújo D. Noção de Competência e Organização Curricular. RBSP. 2007;31(supl. 1):32-43.

28. Lucchese R, Vera I, Pereira WR. As políticas de saúde - SUS - como referência para o processo ensino-aprendizagem do enfermeiro. Rev. Eletr. Enf. 2010;12(3):562-6.

29. Moretti-Pires RO. Complexidade em saúde Família e a Formação do Futuro profissional de saúde. Interface (Botucatu). 2009; 3(30):153-66.

30. Pontes ALM, Bornstein VJ, Giugliani C. O agente comunitário de saúde em Angola: desafios par sua atuação e para formação profissional em saúde. Trab. Ed. Saúde. 2012;9(3):521-33. 
31. Gomes KO, Cotta RMM, Mitre SM, Batista RS, Cherchiglia ML. O agente comunitário de saúde e a consolidação do Sistema Único de Saúde: reflexões contemporâneas. Physis. 20(4):1143-64.

Recebido em: 27/03/2018

Aceito em: 04/06/2018 